\title{
Recommandations pour la pratique clinique en soins de support, Nice, Saint-Paul-de-Vence : et de une !
}

\section{Krakowski}

Service d'oncologie médicale, centre Alexis-Vautrin, avenue de Bourgogne, F-54500 Vandœuvre-lès-Nancy, France

Correspondance : i.krakowski@nancy.fnclcc.fr

Les voici ! Personne n'en a parlé, et elles n'étaient donc pas attendues : elles sont toutefois à votre disposition après un long travail d'élaboration et de relecture qui, je l'espère, répondra à vos préoccupations de terrain.

Cette introduction, en miroir de l'éditorial signé par Moïse Namer et Joseph Gligorov pour la partie I, pour vous dire l'intérêt et l'enrichissement des oncologues et des autres professionnels, médecins ou non, qui se sont investis sur ces thèmes émergents des soins de support. Tous ne sont pas représentés ici, évidemment tant les thèmes à décliner sont nombreux... Tous doivent être remerciés d'avoir relevé le défi de cette "Première ". Un des premiers édito de notre revue sur les soins oncologiques de support avait soulevé cette question : les cancérologues sont-ils concernés par les soins de support? Et la réponse nous était apparue évidente : franchement oui et sans réserves, car il est impossible de déléguer toute la gestion des symptômes propres à la maladie ou aux traitements spécifiques, celle de l'annonce et de l'aide psychologique au moins minimum même si des recours d'experts sont possibles. La présence de 300 oncologues à la première journée soins de support des RPC, à Nice le 14 janvier 2009, est un témoignage supplémentaire de leur envie de réfléchir et d'améliorer leurs pratiques sur ces sujets. Les soins de support, on le voit dans cette revue, concernent tous les malades. Ils permettent de limiter les toxicités et les séquelles de ceux qui vont guérir; de prendre soin de ceux qui vont mourir en cas d'échec de cette médecine " heureusement triomphante ". "L'humanité gémit sous le poids du progrès qu'elle a généré. " disait $H$. Bergson. L'échec, ce n'est pas de perdre le combat du cancer, c'est de mal le combattre ! De bons soins de support sont aujourd'hui la garantie de meilleurs taux de guérison, d'une meilleure qualité de vie, d'une meilleure mort, et aucun de nous ne peut se désintéresser de ces trois attentes, toutes légitimes, de nos malades et de leurs proches... Ou alors... Que soient remerciés ici les fondateurs des RPC, dont l'expérience de femmes et d'hommes de terrain, la culture scientifique et I'humanité ont conduit à demander que l'Association francophone pour les soins oncologiques de support (Afsos) soit partenaire des RPC. À cette occasion : bienvenue au plus grand nombre de lecteurs sur www.afsos.org et aux nouveaux membres de cette société savante que l'on pourrait qualifier de "nouvelle plateforme organique " pour entrer dans les terminologies à la mode... Non, les soins oncologiques de support ne s'improvisent pas; oui, les soins oncologiques de support font partie intégrante de la cancérologie et ont besoin de progresser et de s'organiser en son sein... sans oublier les autres organes! 